\title{
openheart Predicting clinical outcome by indexed mitral valve tenting in functional mitral valve regurgitation
}

\author{
Maria von Stumm (D) , ${ }^{1}$ Florian Dudde, ${ }^{1}$ Theresa Holst, ${ }^{1}$ Tatjana Sequeira-Gross, ${ }^{1}$ \\ Jonas Pausch, ${ }^{1}$ Lisa Müller, ${ }^{1}$ Christoph R Sinning (D) , ${ }^{2}$ Hermann Reichenspurner, ${ }^{1}$ \\ E Girdauskas ${ }^{1}$
}

To cite: von Stumm M, Dudde F, Holst T, et al. Predicting clinical outcome by indexed mitral valve tenting in functional mitral valve regurgitation. Open Heart 2021;8:e001483. doi:10.1136/ openhrt-2020-001483

Received 28 0ctober 2020 Revised 2 December 2020 Accepted 2 December 2020

\section{Check for updates}

\section{C Author(s) (or their} employer(s)) 2021. Re-use permitted under CC BY-NC. No commercial re-use. See rights and permissions. Published by BMJ.

${ }^{1}$ Cardiovascular Surgery, University Hospital Hamburg Eppendorf University Heart Centre, Hamburg, Germany ${ }^{2}$ General and Interventional Cardiology, University Hospital Hamburg Eppendorf University Heart Centre, Hamburg, Germany

\section{Correspondence to} Dr Maria von Stumm; m. stumm@uke.de

\section{ABSTRACT}

Objectives Mitral valve (MV) tenting parameters are indicators of left ventricular remodelling severity and may predict outcome in functional mitral regurgitation (FMR). We hypothesised that indexing of MV tenting area to body surface area (BSA), to mitral annulus diameter or genderadjusted analysis of tenting parameters may improve their prognostic value.

Methods We identified retrospectively 240 patients with consecutive FMR (mean age $68 \pm 10$ years; men $=135$ ) from our institutional database who underwent isolated MV annuloplasty during a period of 7 years (2010-2016). Using preoperative two-dimensional transthoracic echocardiographic images, MV tenting parameters including tenting area, tenting height and annulus diameter were systematically assessed. Follow-up protocol consisted of chart review and structured clinical questionnaire. Primary study endpoint was the composite of death and adverse cardiac events (ie, MV reoperation, cardiac resynchronisation therapy implantation, ventricular assist device implantation or heart transplantation). Results BSA-indexed MV tenting area was identified as independent predictor of primary study endpoint (HR 1.9; $95 \% \mathrm{Cl} 1.1$ to $3.5 ; p=0.02$ ). After cut-off point analysis, BSA-indexed MV tenting area $>1.35 \mathrm{~cm}^{2} / \mathrm{m}^{2}$ was significantly associated with primary study outcome (HR 2.3; $95 \% \mathrm{Cl} 1.3$ to 4.0; $\mathrm{p}=0.003$ ). Annulus-indexed MV tenting area showed only a tendency towards primary study endpoint prediction (HR 2.8; 95\% $\mathrm{Cl} 0.6$ to 12.6 ; $\mathrm{p}=0.17)$. Between female and male patients, BSA-indexed MV tenting area was similar $\left(1.42 \pm 0.4 \mathrm{~cm}^{2} / \mathrm{m}^{2}\right.$ vs $\left.1.45 \pm 0.4 \mathrm{~cm}^{2} / \mathrm{cm}^{2} ; \mathrm{p}=0.6\right)$ and gender was not associated with primary study outcome (HR $0.8 ; 95 \% \mathrm{Cl} 0.5$ to 1.4 ; $\mathrm{p}=0.5$ ).

Conclusion In our FMR cohort, BSA-indexed MV tenting area showed the strongest association with negative outcomes following isolated MV annuloplasty. Patients with BSA-indexed MV tenting area $>1.35 \mathrm{~cm}^{2} / \mathrm{m}^{2}$ could potentially benefit from additional surgical maneuvers addressing left ventricular remodelling.

\section{INTRODUCTION}

Progressive left ventricular remodelling is frequently followed by a functional regurgitation of the structurally normal mitral valve

\section{Key questions}

What is already known about this subject?

- Severity of mitral valve (MV) tenting (ie, MV tenting area $>2.4 \mathrm{~cm}^{2}$ ) was previously shown to correlate with negative outcomes in patients with functional mitral regurgitation (FMR).

What does this study add?

- In our study, we focused on confounding factors of MV tenting area including body shape (BSA), MV geometry (annulus diameter) and gender. We were able to demonstrate that BSA-adjusted MV tenting area $>1.35 \mathrm{~cm}^{2} / \mathrm{m}^{2}$ is a reliable echocardiographic marker predicting reduced survival and lower freedom of adverse cardiac events following isolated MV annuloplasty.

How might this impact on clinical practice?

- Patients with FMR should preoperatively undergo detailed echocardiographic examination with assessment of tenting parameters including BSAindexed tenting area. Surgical treatment strategy of FMR should be adapted according to these measurements. In patients with FMR with a BSA-indexed tenting area $>1.35 \mathrm{~cm}^{2} / \mathrm{m}^{2}$, we strongly recommend performing MV annuloplasty with simultaneous subannular repair.

(FMR). ${ }^{1}$ Due to papillary muscle displacement, increased tethering forces on mitral chordae and annular dilatation, mitral valve (MV) leaflets lose their coaptation and take shape of a tent during systole. ${ }^{2}$ Typically, the triangular area between mitral annulus and tented leaflets is described as MV tenting area. ${ }^{3}$ MV tenting area can be quantitatively assessed by echocardiography and reflects both the global and regional left ventricular remodelling. ${ }^{34}$ Severity of MV tenting has been shown to correlate with prognosis of patients with FMR ${ }^{5-9}$ Recurrent mitral regurgitation (MR), heart failure and death occurred more often in patients with FMR with a preoperative MV tenting area $>2.5 \mathrm{~cm}^{2}{ }^{5-9}$ Though, it is difficult 
A

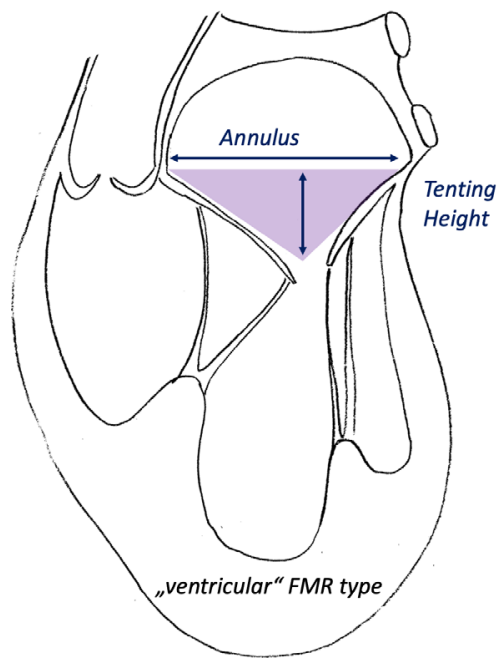

B

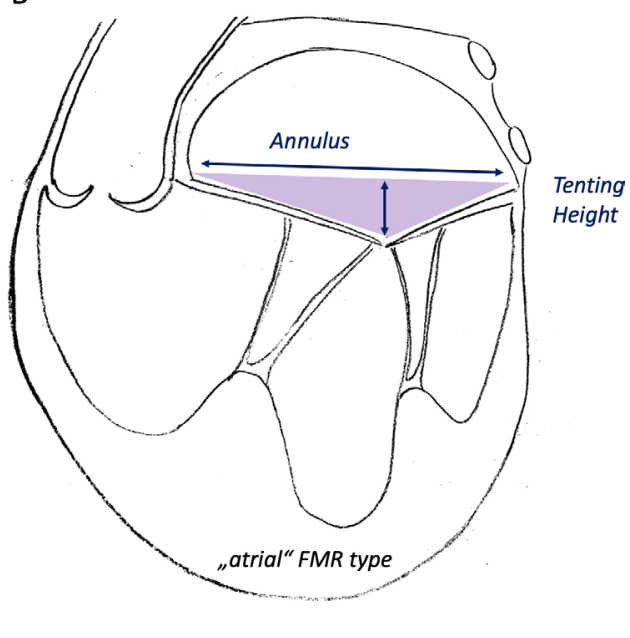

Figure 1 Illustration of individual variations of tenting area (purple triangle), annulus diameter and left ventricular remodelling. A mitral valve tenting area of $3.0 \mathrm{~cm}^{2}$ can be found in patients with severe left ventricular remodelling (annulus diameter $40 \mathrm{~mm}$; tenting height $15 \mathrm{~mm}$ ) (A) and in patients with severe annular dilatation (annulus diameter $60 \mathrm{~mm}$; tenting height $10 \mathrm{~mm}$ ) (B). Therefore, 'absolute' tenting area does not reflect on annular dilatation. FMR, functional mitral regurgitation.

to interpret MV tenting area without looking at potential confounding factors: (1) MV tenting area does not consider the variance in body shape of patients with FMR. Larger individuals have normally larger heart size and, therefore, larger absolute tenting area. ${ }^{10}$ (2) MV tenting area does not adjust for MV annulus diameter, which may vary significantly among individuals with different forms of FMR ('atrial vs ventricular FMR type; figure 1). ${ }^{11}$ (3) Absolute MV tenting area does not reflect potential gender differences. Female patients are known to have smaller left ventricle (LV) volumes in FMR and still experience higher mortality in comparison to men. ${ }^{12}{ }^{13}$ Therefore, adjustment of tenting parameters to the individual anatomy seems to be reasonable and could improve the predictive value of tenting parameters to enable an individualised treatment strategy ${ }^{14}$ However, the evidence of individualised imaging markers (ie, indexed to body surface area (BSA) or sex) in FMR is very sparse. Herewith, we assessed preoperative MV tenting area in patients with FMR and adjusted MV tenting area to BSA, mitral annulus diameter and gender. We hypothesised that severity of indexed MV tenting is associated with the outcome after MV surgery in patients with FMR. Second, we aimed to identify those patients with FMR, who are not sufficiently treated by an isolated mitral annuloplasty and could potentially benefit from modified surgical strategy (ie, subannular MV repair).

\section{METHODS}

\section{Study design}

In our study, we retrospectively reviewed 1661 patients from our institutional MV database who underwent MV surgery for MV regurgitation from January 2010 to December 2016. Only patients with FMR disease $(\mathrm{n}=521)$ were included in our analysis (figure 2). Further patients were excluded $(n=259)$ due to incorrect storage of echocardiographic images or insufficient image quality to measure tenting parameters. Finally, a total of 240 patients with FMR who underwent an isolated MV annuloplasty (ie, without additional leaflet manoeuvres or subannular repair) served as our study population. We included most recent long-term follow-up data with a mean follow-up period for the whole study cohort of $43 \pm 25$ months. Preliminary results with a limited follow-up had been previously published by our group. ${ }^{5}$

Individual patient consent was waived. Patients or public were not involved in our study design.

\section{Echocardiographic assessment of tenting parameter}

Preoperative two-dimensional (2D) transthoracic echocardiography was routinely performed in our echocardiographic core laboratory within 1 week prior to surgery. Echocardiographic images were systematically analysed by two independent investigators ( $\mathrm{MvS}$ and TS-G). Using a standardised protocol, MV tenting was assessed in the parasternal-long axis view during late systole according to the recommendations for the assessment of valvular regurgitation of the European Association of Echocardiography. ${ }^{3}$ The extent of MV tenting was assessed by tenting area $\left(\mathrm{cm}^{2}\right)$ and tenting height $(\mathrm{mm})$ (figure 3). Annulus diameter was also measured in the parasternallong axis view during late systole representing the anteroposterior diameter.

\section{Surgical mitral annuloplasty}

Surgical approach to FMR was either median sternotomy or right anterolateral mini-thoracotomy, depending on concomitant cardiac procedures and patients' comorbidities. FMR was treated using a standard rigid or semirigid complete annuloplasty ring, which was downsized by one size, according to the length of the anterior mitral leaflet. There were no major variations in the surgical 


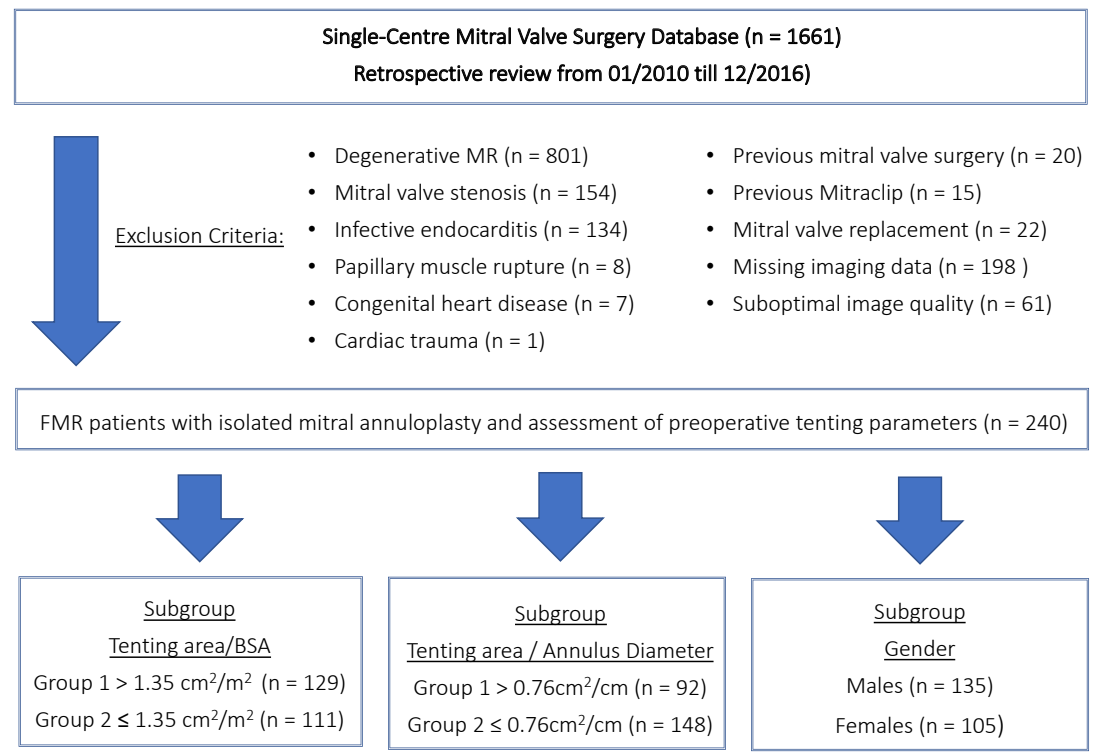

Figure 2 Flow chart of study design and patient selection. BSA, body surface area; FMR, functional mitral regurgitation; MR, mitral regurgitation.

management and perioperative treatment protocol during the study period. Baseline heart failure medication was maintained during the perioperative period and included aspirin, lipid-lowering agents, beta-blockers, ACE inhibitors and diuretics.

\section{Clinical outcome and follow-up}

Clinical outcomes of interest were defined as all-cause mortality and adverse cardiac events, which consisted of MV reoperation, cardiac resynchronisation therapy (CRT) implantation, ventricular assist device (VAD) implantation or heart transplantation. Primary study endpoint was a composite of death and adverse cardiac events.

All patients underwent regularly scheduled follow-up visits in outpatient heart failure units and their medical records were analysed. Furthermore, all patients were contacted by a telephone interview using a standardised clinical outcome questionnaire.

\section{Statistical analysis}

Normally distributed continuous variables are presented as mean $\pm \mathrm{SD}$ and categorical variables are expressed as percentages throughout the manuscript. Comparison of normally distributed continuous variables was performed by unpaired t-test. Fisher's exact test was used for univariable comparisons of categorical variables. A Pearson correlation coefficient was used for correlation analyses.

Overall survival and freedom of adverse cardiac events in study groups were compared using univariable logrank test (Kaplan-Meier method). Predictors of primary endpoint variable were subsequently assessed by multivariable Cox regression analysis. All $\mathrm{p}$ values $<0.05$ were considered statistically significant. All statistical analyses
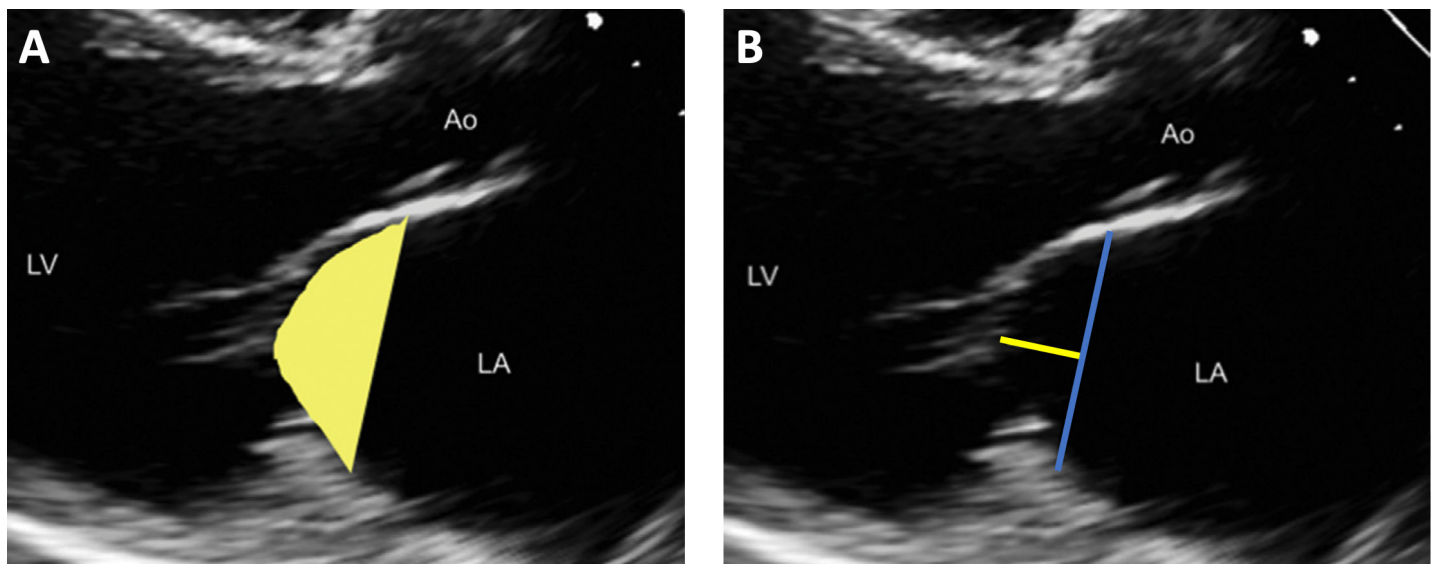

Figure 3 Measurement of tenting parameter by transthoracic two-dimensional echocardiography in the parasternal-long axis view during late systole: (A) tenting area (yellow area), (B) tenting height (yellow line) and annulus diameter (blue line). Ao, Aorta; LA, left atrium; LV, left ventricle. 
Table 1 Patient demographics and baseline characteristics

\begin{tabular}{|c|c|c|c|c|c|c|c|c|c|c|}
\hline \multirow[b]{2}{*}{$\begin{array}{l}\text { Patient } \\
\text { characteristics }\end{array}$} & \multirow[b]{2}{*}{$\begin{array}{l}\text { All } \\
(n=240)\end{array}$} & \multicolumn{3}{|c|}{ Tenting area/BSA } & \multicolumn{3}{|c|}{ Tenting area/annulus diameter } & \multicolumn{3}{|c|}{ Gender differences } \\
\hline & & $\begin{array}{l}>1.35 \mathrm{~cm}^{2} / \\
\mathrm{m}^{2} \\
(\mathrm{n}=129)\end{array}$ & $\begin{array}{l}\leq 1.35 \mathrm{~cm}^{2} / \\
\mathrm{m}^{2} \\
(\mathrm{n}=111)\end{array}$ & $P$ value & $\begin{array}{l}>0.76 \mathrm{~cm}^{2} / \\
\mathrm{cm} \\
(\mathrm{n}=92)\end{array}$ & $\begin{array}{l}\leq 0.76 \mathrm{~cm}^{2} / \\
\mathrm{cm} \\
(\mathrm{n}=148)\end{array}$ & $P$ value & $\begin{array}{l}\text { Females } \\
(n=105)\end{array}$ & $\begin{array}{l}\text { Males } \\
(n=135)\end{array}$ & $P$ value \\
\hline Male (\%) & $135(56.3)$ & $72(55.8)$ & $63(56.8)$ & 0.88 & $53(55.4)$ & $82(57.6)$ & 0.74 & - & - & - \\
\hline Age (years) & $68.0 \pm 9.8$ & $67.5 \pm 9.8$ & $68.4 \pm 9.7$ & 0.59 & $67.1 \pm 10.1$ & $68.4 \pm 4.3$ & 0.33 & $68.1 \pm 9.8$ & $67.2 \pm 8.2$ & 0.17 \\
\hline $\mathrm{BSA}\left(\mathrm{m}^{2}\right)$ & $1.9 \pm 0.2$ & $1.9 \pm 0.2$ & $2.0 \pm 0.2$ & 0.04 & $2.0 \pm 0.2$ & $1.9 \pm 0.2$ & 0.37 & $1.8 \pm 0.2$ & $2.0 \pm 0.1$ & $<0.001$ \\
\hline Diabetes (\%) & $44(18.3)$ & $24(18.2)$ & $20(18.6)$ & 0.93 & $18(17.7)$ & $26(19.6)$ & 0.72 & $16(15.2)$ & $28(20.9)$ & 0.24 \\
\hline Creatinine (mg/dL) & $1.3 \pm 0.8$ & $1.4 \pm 1.0$ & $1.2 \pm 0.6$ & 0.11 & $1.4 \pm 1.0$ & $1.3 \pm 0.7$ & 0.31 & $1.3 \pm 0.5$ & $1.3 \pm 0.7$ & 0.96 \\
\hline CAD (\%) & $127(52.9)$ & $66(51.6)$ & $61(55.0)$ & 0.56 & $51(55.4)$ & $76(51.4)$ & 0.54 & $37(35.2)$ & $90(66.7)$ & $<0.001$ \\
\hline Acute HF (\%) & $107(44.6)$ & $66(51.6)$ & $41(36.9)$ & 0.02 & $45(49.5)$ & $62(41.9)$ & 0.25 & $46(43.8)$ & $61(45.5)$ & 0.79 \\
\hline $\operatorname{LVEF}(\%)$ & $46 \pm 12$ & $44 \pm 12$ & $49 \pm 12$ & 0.01 & $43 \pm 13$ & $48 \pm 12$ & 0.04 & $49 \pm 11$ & $44 \pm 13$ & 0.04 \\
\hline Ischaemic CM & $135(56.3)$ & $73(56.6)$ & $62(55.9)$ & 0.91 & $78(52.7)$ & $57(62)$ & 0.16 & $42(40)$ & $93(68.9)$ & $<0.001$ \\
\hline Non-ischaemic CM & $105(43.7)$ & $56(43.4)$ & $49(44.1)$ & 0.91 & $70(47.3)$ & $35(38)$ & 0.16 & $63(60)$ & $42(31.1)$ & $<0.001$ \\
\hline STS Score (\%) & $3.4 \pm 3.5$ & $3.5 \pm 3.9$ & $3.2 \pm 3.0$ & 0.58 & $3.2 \pm 2.4$ & $3.4 \pm 4.0$ & 0.68 & $3.6 \pm 4.5$ & $3.2 \pm 3.5$ & 0.33 \\
\hline EuroSCORE II (\%) & $7.1 \pm 8.9$ & $7.0 \pm 8.5$ & $7.3 \pm 9.4$ & 0.83 & $7.5 \pm 8.9$ & $6.9 \pm 8.9$ & 0.65 & $7.4 \pm 9.9$ & $7.0 \pm 8.2$ & 0.71 \\
\hline Ring size (mm) & $29.9 \pm 1.6$ & $29.9 \pm 1.8$ & $30.0 \pm 1.7$ & 0.69 & $30.0 \pm 1.8$ & $29.9 \pm 1.7$ & 0.64 & $29.3 \pm 1.6$ & $30.5 \pm 1.7$ & $<0.001$ \\
\hline Concomit. proc. (\%) & $184(76.7)$ & $97(75.2)$ & 87 (78.4) & 0.56 & $67(79.1)$ & $117(72.8)$ & 0.76 & $71(67.6)$ & 113 (83.7) & 0.003 \\
\hline CABG (\%) & 94 (39.2) & 41 (31.8) & $53(47.7)$ & 0.01 & $33(35.9)$ & $61(41.2)$ & 0.41 & $23(21.9)$ & 71 (52.6) & $<0.001$ \\
\hline
\end{tabular}

Continuous values presented means \pm SD; categorial values presented as no. (\%).

Acute HF, decompensated heart failure within two weeks before surgery; BSA, body surface area $\left(\mathrm{m}^{2}\right)$; $\mathrm{CABG}$, coronary artery bypass grafting; CAD, Coronary artery disease; CM, cardiomyopathy; LVEF, left ventricle ejection fraction; STS Score, Society of Thoracic Surgery risk score for mortality.

were performed using the SPSS V.26.0 statistical package (IBM Corp.).

\section{Post-hoc analyses of BSA-indexed, annulus-indexed and gender-indexed MV tenting area}

Tenting area was indexed to BSA and annular diameter by simple division of variables without additional weighting factors. BSA $\left(\mathrm{m}^{2}\right)$ was calculated using the formula of Mosteller. ${ }^{16}$ To investigate associations between tenting area versus BSA and tenting area versus annular diameter we performed bivariate (Pearson) correlations. To demonstrate associations between gender and tenting area, we used a $\chi^{2}$ test. For prognostic assessment of indexed tenting area and gender on clinical outcome, multivariable analyses were performed for each parameter using a Cox proportional hazards regression model of six covariates, which showed $\mathrm{p}<0.1$ in the previous univariable analysis or were clinically relevant. In addition, we calculated receiver operating characteristic (ROC) curves to determine optimal cut-off points of BSA and annulus-indexed tenting area to predict primary study endpoint. The optimal cut-off value was defined by Youden index.

\section{RESULTS \\ Demographics and baseline characteristics}

A total of 240 consecutive patients (mean age 68.0 \pm 9.8 years, 135 men) were analysed. Ischaemic cardiomyopathy was found in 135 (56.3\%) patients, the remaining 105 patients had a non-ischaemic cardiomyopathy. Risk score calculations revealed mean logistic EuroSCORE II of $7.1 \% \pm 8.9 \%$ and Society of Thoracic Surgery Score of $3.4 \% \pm 3.5 \%$, respectively. Isolated MV annuloplasty was performed in 58 (23\%) patients with FMR, while 184 $(77 \%)$ patients underwent concomitant procedures (ie,

Table 2 Preoperative tenting parameters (presented as mean values and separated by gender)

\begin{tabular}{lcccc}
\hline Tenting parameter & All $(\mathbf{n}=\mathbf{2 4 0})$ & Males $(\mathbf{n = 1 3 5 )}$ & Females $(\mathbf{n}=105)$ & P value \\
\hline Annulus diameter $(\mathrm{mm})$ & $37.3 \pm 5.4$ & $38.6 \pm 5.5$ & $36.2 \pm 5.1$ & 0.001 \\
BSA-adjusted annulus diameter $\left(\mathrm{cm} / \mathrm{m}^{2}\right)$ & $19.4 \pm 3.0$ & $18.8 \pm 2.8$ & $20.1 \pm 3.1$ & 0.003 \\
Tenting area $\left(\mathrm{cm}^{2}\right)$ & $2.8 \pm 0.8$ & $2.9 \pm 0.8$ & $2.6 \pm 0.7$ & 0.017 \\
BSA-adjusted tenting area $\left(\mathrm{cm}^{2} / \mathrm{m}^{2}\right)$ & $1.43 \pm 0.4$ & $1.42 \pm 0.4$ & $1.45 \pm 0.4$ & 0.58 \\
Tenting height $(\mathrm{mm})$ & $11.4 \pm 2.8$ & $11.9 \pm 2.9$ & $10.9 \pm 2.6$ & 0.009 \\
BSA-adjusted tenting height $\left(\mathrm{mm} / \mathrm{m}^{2}\right)$ & $6.0 \pm 1.5$ & $5.9 \pm 1.6$ & $6.1 \pm 1.5$ & 0.315 \\
\hline
\end{tabular}

Continuous values presented means \pm SD

BSA, body surface area. 
Table 3 Predictors of the composite of survival and freedom of cardiac adverse events

\begin{tabular}{|c|c|c|c|c|}
\hline & B & $P$ value & HR & Cl \\
\hline \multicolumn{5}{|c|}{ Impact of BSA-adjusted tenting area } \\
\hline Age & 0.00 & 0.98 & 1.00 & 0.97 to 1.03 \\
\hline Log. EuroScore II & 0.02 & 0.04 & 1.02 & 0.99 to 1.05 \\
\hline Coronary disease & 0.68 & 0.02 & 1.97 & 1.14 to 3.40 \\
\hline Creatinine level & 0.10 & 0.44 & 1.10 & 0.86 to 1.43 \\
\hline LVEF & -0.03 & 0.01 & 0.97 & 0.95 to 0.99 \\
\hline Tenting area/BSA* & 0.67 & 0.02 & 1.94 & 1.09 to 3.47 \\
\hline \multicolumn{5}{|c|}{ Impact of BSA-adjusted tenting area $>1.35 \mathrm{~cm}^{2} / \mathrm{m}^{2}$} \\
\hline Age & -0.02 & 0.87 & 0.99 & 0.97 to 1.03 \\
\hline Log. EuroScore II & 0.02 & 0.10 & 1.02 & 1.00 to 1.05 \\
\hline Coronary disease & 0.69 & 0.01 & 2.00 & 1.16 to 3.41 \\
\hline Creatinine level & 0.08 & 0.56 & 1.08 & 0.83 to 1.40 \\
\hline LVEF & -0.03 & 0.01 & 0.97 & 0.95 to 0.99 \\
\hline $\begin{array}{l}\text { Tenting area/BSA } \\
>1.35 \mathrm{~cm}^{2} / \mathrm{m}^{2} \dagger\end{array}$ & 0.84 & 0.003 & 2.31 & 1.32 to 4.04 \\
\hline \multicolumn{5}{|c|}{ Impact of annulus-adjusted tenting area } \\
\hline Age & 0.00 & 0.99 & 1.00 & 0.97 to 1.03 \\
\hline Log. EuroScore II & 0.02 & 0.19 & 1.02 & 0.99 to 1.05 \\
\hline Coronary disease & 0.58 & 0.03 & 1.79 & 1.05 to 3.06 \\
\hline Creatinine level & 0.12 & 0.37 & 1.12 & 0.87 to 1.45 \\
\hline LVEF & -0.03 & 0.004 & 0.97 & 0.95 to 0.99 \\
\hline Tenting area/annulus* & 1.04 & 0.17 & 2.84 & 0.64 to 12.6 \\
\hline \multicolumn{5}{|c|}{ Impact of annulus-adjusted tenting area $>0.76 \mathrm{~cm}^{2} / \mathrm{cm}$} \\
\hline Age & -0.01 & 0.96 & 1.00 & 0.97 to 1.03 \\
\hline Log. EuroScore II & 0.02 & 0.17 & 1.02 & 0.99 to 1.05 \\
\hline Coronary disease & 0.58 & 0.03 & 1.79 & 1.05 to 3.06 \\
\hline Creatinine level & 0.09 & 0.47 & 1.10 & 0.85 to 1.42 \\
\hline LVEF & -0.03 & 0.01 & 0.97 & 0.95 to 0.99 \\
\hline $\begin{array}{l}\text { Tenting area/annulus } \\
>0.76 \mathrm{~cm}^{2} / \mathrm{cm} \dagger\end{array}$ & 0.55 & 0.037 & 1.72 & 1.03 to 2.89 \\
\hline \multicolumn{5}{|l|}{ Impact of gender } \\
\hline Age & -0.01 & 0.93 & 0.99 & 0.97 to 1.03 \\
\hline Log. EuroScore II & 0.02 & 0.21 & 1.02 & 0.99 to 1.04 \\
\hline Coronary disease & 0.50 & 0.08 & 1.65 & 0.95 to 2.87 \\
\hline Creatinine level & 0.12 & 0.38 & 1.12 & 0.87 to 1.45 \\
\hline LVEF & -0.03 & 0.003 & 0.97 & 0.95 to 0.99 \\
\hline Female sex & -0.18 & 0.51 & 0.83 & 0.48 to 1.44 \\
\hline
\end{tabular}

Independent predictors of the composite of survival and freedom from adverse cardiac events were assessed by multivariable analysis using the Cox proportional hazards regression model.

${ }^{*}$ Continuous variable.

†Dichotomised variable.

BSA, body surface area; LVEF, left ventricle ejection fraction.

coronary artery bypass grafting (CABG), tricuspid valve surgery, aortic valve surgery; table 1 ).

Preoperative assessment of echocardiographic MV tenting parameters revealed mean tenting area of $2.8 \pm 0.8 \mathrm{~cm}^{2}$ and mean anteroposterior annulus diameter of $3.7 \pm 0.5 \mathrm{~cm}$. Mean BSA was $1.9 \pm 0.2 \mathrm{~m}^{2}$ (table 2).
In the whole study cohort, $51 / 240(21.2 \%)$ patients died and 14/240 (5.8\%) patients experienced an adverse cardiac event including MV reoperation $(n=6)$, CRT implantation $(n=4)$, VAD implantation $(n=3)$ and heart transplantation $(n=1)$ during follow-up. Calculated by Kaplan-Meier analysis, overall cardiac adverse event free survival was $70.8 \% \pm 3.4 \%$ at 5 years in the whole study cohort.

\section{BSA-indexed MV tenting area}

We found a significant correlation between tenting area and BSA (Pearson $\mathrm{r}=0.216 ; \mathrm{p}=0.01$ ). Indexation of tenting area to BSA revealed a normally distributed variable with a mean value of $1.43 \pm 0.4 \mathrm{~cm}^{2} / \mathrm{m}^{2}$ (minimum $0.57 \mathrm{~cm}^{2} /$ $\mathrm{m}^{2}$-maximum $2.67 \mathrm{~cm}^{2} / \mathrm{m}^{2}$ ). In the multivariable analysis, BSA-indexed MV tenting area was identified as independent predictor of composite primary endpoint (HR 1.9; 95\% CI 1.1 to 3.5; $\mathrm{p}=0.02$; table 3). Optimal cut-off point of BSA-indexed MV tenting area, as identified by ROC analysis, was $1.35 \mathrm{~cm}^{2} / \mathrm{m}^{2}$. Subsequently, we subdivided our study cohort into 111 patients with a BSA-indexed MV tenting area $\leq 1.35 \mathrm{~cm}^{2} / \mathrm{m}^{2}$ and 129 patients with a BSAindexed MV tenting area $>1.35 \mathrm{~cm}^{2} / \mathrm{m}^{2}$. As expected, baseline characteristics of both subgroups revealed significant differences (table 1). Those patients with BSA-indexed MV tenting area $>1.35 \mathrm{~cm}^{2} / \mathrm{m}^{2}$ presented with a worse baseline left ventricle ejection fraction (LVEF), had more often decompensated heart failure (ie, cardiac decompensation within 2 weeks before surgery) and underwent less concomitant CABG surgery. Overall, 44/129 (34.1\%) patients with BSA-indexed MV tenting area $>1.35 \mathrm{~cm}^{2} / \mathrm{m}^{2}$ died or had an adverse cardiac event during the follow-up period versus 21/111 (18.9\%) patients with BSA-indexed MV tenting area $\leq 1.35 \mathrm{~cm}^{2} / \mathrm{m}^{2}$. In Kaplan-Meier analysis, BSA-indexed MV tenting area $>1.35 \mathrm{~cm}^{2} / \mathrm{m}^{2}$ was significantly associated with the primary study outcome $\left(\mathrm{p}_{\text {(log-rank) }}=0.002\right)$ (figure $\left.4 \mathrm{~A}\right)$. In addition, multivariable analysis revealed that patients with a BSA-indexed MV tenting area $>1.35 \mathrm{~cm}^{2} / \mathrm{m}^{2}$ had a 2.3 times greater risk for experiencing primary study endpoint (HR 2.3; 95\% CI 1.3 to 4.0; $\mathrm{p}=0.003$; table 3 ).

\section{Tenting area indexed by annulus diameter}

MV tenting area showed a strong correlation with the anteroposterior annulus diameter (Pearson $r=0.663$, $\mathrm{p}<0.0001)$. Indexation of tenting area to annulus diameter revealed a normally distributed variable with a mean value of $0.74 \mathrm{~cm}^{2} / \mathrm{cm}$ (minimum $0.34 \mathrm{~cm}^{2} / \mathrm{m}^{2}-$ maximum $1.31 \mathrm{~cm}^{2} / \mathrm{cm}$ ). Annulus-indexed MV tenting area showed only a tendency towards an association with primary study endpoint in the multivariable analysis (HR 2.8; $95 \%$ CI 0.6 to 12.6 ; $\mathrm{p}=0.17$; table 3 ). Optimal cut-off point of annulus-indexed MV tenting area, as identified by ROC analysis, was $0.76 \mathrm{~cm}^{2} / \mathrm{cm}$. We subdivided our study cohort into 148 patients with annulus-indexed MV tenting area $\leq 0.76 \mathrm{~cm}^{2} / \mathrm{cm}$ and 92 patients with annulusindexed MV tenting area $>0.76 \mathrm{~cm}^{2} / \mathrm{cm}$. Both subgroups had comparable baseline characteristics (table 1). 

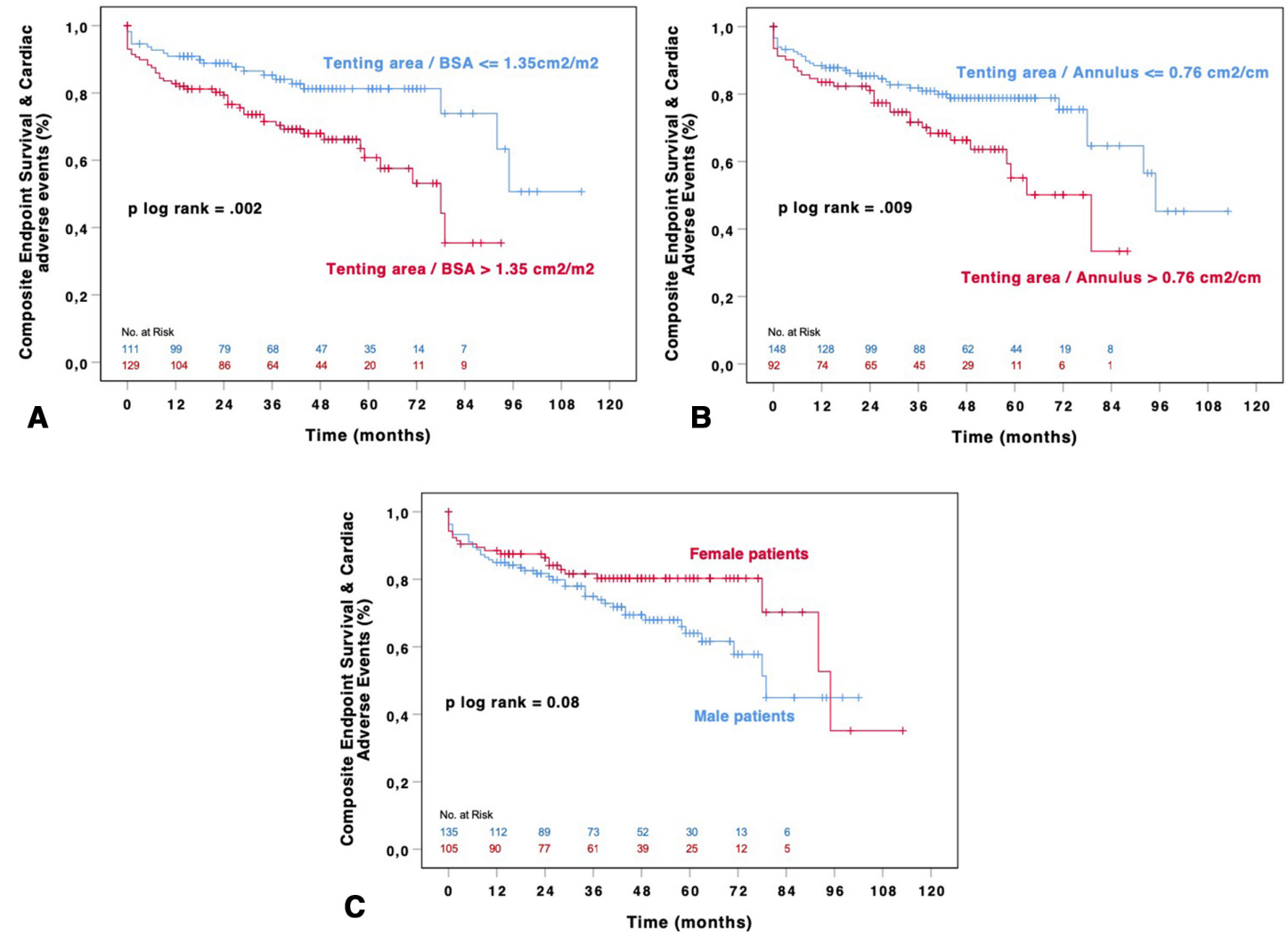

Figure 4 (A) Univariate Kaplan-Meier analysis of the composite of survival and freedom of cardiac adverse events using tenting area adjusted for body surface area (cut-off point of $1.35 \mathrm{~cm}^{2} / \mathrm{m}^{2}$ ) to divide the study cohort. (B) Univariate KaplanMeier analysis of the composite of survival and freedom of cardiac adverse events using the tenting area adjusted for annulus diameter (cut-off point of $0.76 \mathrm{~cm}^{2} / \mathrm{cm}$ ) to divide the study cohort. (C) Univariate Kaplan-Meier analysis of the composite of survival and freedom of cardiac adverse events using gender for subgrouping. BSA, body surface area.

During the follow-up period, 32/92 $(34.8 \%)$ patients with annulus-indexed MV tenting area $>0.76 \mathrm{~cm}^{2} / \mathrm{cm}$ died or had an adverse cardiac event versus 33/148 $(22.3 \%)$ patients with annulus-indexed MV tenting area $\leq 0.76 \mathrm{~cm}^{2} / \mathrm{cm}$. Calculated by Kaplan-Meier analysis, annulus-indexed MV tenting area $>0.76 \mathrm{~cm}^{2} / \mathrm{cm}$ was significantly associated with the primary study outcome $\left(\mathrm{p}_{\text {(log-rank })}=0.009\right)$ (figure $\left.4 \mathrm{~B}\right)$. In addition, multivariable analysis revealed that patients with annulus-indexed MV tenting area $>0.76 \mathrm{~cm}^{2} / \mathrm{cm}$ had a 1.7 times greater risk for experiencing primary study endpoint (HR 1.7; 95\% CI 1.0 to $2.9 ; \mathrm{p}=0.037$ ) (table 3 ).

\section{Subgrouping by gender}

Our study cohort included 105 (44\%) female and 135 $(56 \%)$ male patients. Comparison of baseline characteristics between female and male patients revealed significantly lower BSA values and lower prevalence of coronary artery disease (CAD) in women. Consequently, women underwent less concomitant CABG surgery. Furthermore, smaller annuloplasty rings were implanted in female patients (table 1). The comparison of tenting parameters between female and male patients revealed a significantly lower MV tenting area, tenting height and annulus diameter in women (table 2). After adjustment to BSA, mean values of BSA-indexed tenting area and BSA-indexed tenting height were comparable between female and male patients. Gender was not significantly associated with the primary study endpoint in the Kaplan-Meier analysis $\left(\mathrm{p}_{(\log -}\right.$ rank) $=0.08$ ) (figure 4c) and in the multivariable Cox regression analysis (HR $0.8 ; 95 \%$ CI 0.5 to $1.4 ; \mathrm{p}=0.5$ ).

\section{DISCUSSION}

Herewith, we aimed to evaluate the relationship between severity of preoperative MV tenting area-indexed to BSA, mitral annulus diameter or separated by gender-and adverse clinical outcome in patients with FMR who underwent an isolated MV annuloplasty. Each indexing factor correlated significantly with tenting area. BSA-adjusted MV tenting area showed the strongest association with clinical outcomes in our cohort. BSA-adjusted MV tenting area $>1.35 \mathrm{~cm}^{2} / \mathrm{m}^{2}$ was associated with reduced survival and lower freedom of adverse cardiac events following isolated MV annuloplasty. Based on these findings, we can confirm our initial hypothesis that the severity of indexed MV tenting area is associated with clinical outcome in FMR.

\section{Further evidence for the predictive value of tenting parameters}

Use of indexed MV tenting parameters is limited and their predictive value in FMR is still insufficiently defined. So far, only two studies addressed BSA-indexed or gender-indexed values of MV tenting area. ${ }^{13}{ }^{17}$ In the former study, published in 2014 by Dwivedi et al, mitral 
Table 4 Published mean values of tenting area in patients with functional mitral regurgitation (FMR) and non-FMR

\begin{tabular}{|c|c|c|c|c|c|c|}
\hline Study & Design & Imaging tool & Population & Parameter & Mean value & $\begin{array}{l}\text { Cut-off } \\
\text { value to } \\
\text { predict } \\
\text { outcome }\end{array}$ \\
\hline \multirow[t]{3}{*}{ Magne et $a^{\beta}$} & \multirow{3}{*}{$\begin{array}{l}\text { Retrospective } \\
\text { observational study }\end{array}$} & \multirow[t]{3}{*}{ 2D Echocardiography } & Non-FMR $(n=20)$ & \multirow[t]{3}{*}{ Tenting area $\left(\mathrm{cm}^{2}\right)$} & $0.95 \pm 0.2$ & \multirow[t]{3}{*}{$2.5 \mathrm{~cm}^{2}$} \\
\hline & & & $\begin{array}{l}\text { Non-persistent MR } \\
(\mathrm{n}=40)\end{array}$ & & $1.72 \pm 0.4$ & \\
\hline & & & Persistent MR $(n=11)$ & & $2.70 \pm 0.9$ & \\
\hline \multirow[t]{2}{*}{ Karaca et $a^{7}$} & \multirow{2}{*}{$\begin{array}{l}\text { Prospective } \\
\text { observational study }\end{array}$} & \multirow[t]{2}{*}{ 2D Echocardiography } & Non-FMR $(n=49)$ & \multirow[t]{2}{*}{ Tenting area $\left(\mathrm{cm}^{2}\right)$} & No values & \multirow[t]{2}{*}{$3.4 \mathrm{~cm}^{2}$} \\
\hline & & & Severe FMR $(n=41)$ & & No values & \\
\hline \multirow[t]{2}{*}{ Dwivedi et $a^{13}$} & \multirow[t]{2}{*}{$\begin{array}{l}\text { Population-based } \\
\text { prospective study }\end{array}$} & \multirow[t]{2}{*}{ 2D Echocardiography } & \multirow[t]{2}{*}{$\begin{array}{l}\text { Healthy subjects } \\
(n=480)\end{array}$} & Tenting area $\left(\mathrm{cm}^{2}\right)$ & $\begin{array}{l}\text { Male: } 2.02 \pm 0.68 \\
\text { Female: } 1.64 \pm 0.47\end{array}$ & \\
\hline & & & & $\begin{array}{l}\text { Indexed tenting } \\
\text { area }\left(\mathrm{cm}^{2} / \mathrm{m}^{2}\right)\end{array}$ & $\begin{array}{l}\text { Male: } 1.02 \pm 0.34 \\
\text { Female: } 0.93 \pm 0.27\end{array}$ & \\
\hline \multirow[t]{3}{*}{ Nishino et al ${ }^{17}$} & \multirow{3}{*}{$\begin{array}{l}\text { Prospective } \\
\text { observational study }\end{array}$} & \multirow[t]{3}{*}{ 2D Echocardiography } & Non-FMR $(n=14)$ & \multirow{3}{*}{$\begin{array}{l}\text { Indexed tenting } \\
\text { area }\left(\mathrm{cm}^{2} / \mathrm{m}^{2}\right)\end{array}$} & $0.42(0.37-0.44)$ & \\
\hline & & & Acute FMR $(\mathrm{n}=44)$ & & $1.02(0.82-1.15)$ & \\
\hline & & & Chronic FMR $(n=36)$ & & $1.56(1.28-1.77)$ & \\
\hline Nappi et $a \beta^{\beta}$ & $\begin{array}{l}\text { Post hoc analysis } \\
\text { of prior published } \\
\text { randomised trial }\end{array}$ & 2D Echocardiography & Severe FMR $(n=48)$ & Tenting area $\left(\mathrm{cm}^{2}\right)$ & $3.0 \pm 0.3$ & $3.1 \mathrm{~cm}^{2}$ \\
\hline Von Stumm et a $\rho^{\Gamma}$ & $\begin{array}{l}\text { Retrospective } \\
\text { observational study }\end{array}$ & 2D Echocardiography & Severe FMR $(n=240)$ & Tenting area $\left(\mathrm{cm}^{2}\right)$ & $2.8 \pm 0.8$ & $2.4 \mathrm{~cm}^{2}$ \\
\hline
\end{tabular}

2D, two-dimensional; MR, mitral regurgitation.

annular dimensions were analysed in healthy subjects. ${ }^{13}$ Average values of BSA-indexed MV tenting area were 1.02 $\mathrm{cm}^{2} / \mathrm{m}^{2}$ in men and $0.93 \mathrm{~cm}^{2} / \mathrm{m}^{2}$ in women. The latter study, published by Nishino $e t$ al, investigated echocardiographic differences between acute and chronic FMR. ${ }^{17}$ In their cohort of patients with chronic FMR, BSA-indexed tenting area varied from 1.3 to $1.8 \mathrm{~cm}^{2} / \mathrm{m}^{2}$. In our cohort, mean values of BSA-adjusted tenting area were $1.42 \pm 0.4 \mathrm{~cm}^{2} / \mathrm{m}^{2}$ in male patients and $1.45 \pm 0.4 \mathrm{~cm}^{2} / \mathrm{m}^{2}$ in female patients, which correlate well with the previously published data in the context of severe FMR. (table 4).

Indexation of MV tenting area to annulus diameter is quite new and we were unable to find any prior data on this topic. Of note, MV tenting area correlated strongly with the anteroposterior MV annulus diameter in our study. However, annulus-indexed MV tenting area (ie, continuous variable) showed only a tendency towards the primary endpoint prediction in the multivariable analysis. We assume that anteroposterior MV annulus measurements, as performed in our study, have only a limited value to describe the entire MV annular geometry. Given the fact that anteroposterior MV annulus measurements did not respect intercommissural annulus size and the saddle shape contour of mitral annulus, the true MV annular dimensions may be underestimated. ${ }^{18}$

The impact of gender in MV disease has been widely evaluated. ${ }^{12}{ }^{19}$ Just recently, a randomised trial by Giustino et al assessed sex-based differences in outcomes after MV surgery for severe FMR. ${ }^{12}$ The authors found significantly lower echocardiographic values of left ventricular diameters (ie, left ventricular end-diastolic diameter, left ventricular end-diastolic volume) and MV apparatus (ie, tethering area, mitral annular area) in women. Furthermore, women with FMR experienced significantly higher mortality and lower quality of life after MV surgery. Our current study revealed smaller echocardiographic dimensions of MV tenting area, anteroposterior MV annulus size and MV tenting height in women which is in accordance with Giustino's study. However, there was no difference in BSA-adjusted MV tenting parameters between both sexes (table 2). Furthermore, we found no sex-related differences in the clinical outcome after MV repair. However, this outcome comparison is blurred by different baseline characteristics men versus women, as demonstrated by better baseline LVEF and lower prevalence of CAD requiring simultaneous CABG in women.

In contrast to a novel concept of indexed MV tenting area, the impact of absolute (ie, non-indexed) MV tenting area in FMR has been extensively analysed in previous studies. Our preliminary study showed that nonindexed MV tenting area $>2.4 \mathrm{~cm}^{2}$ was associated with a worse prognosis in patients with FMR following MV repair (HR 2.1; $\mathrm{p}=0.03$ ). ${ }^{5}$ Other researchers investigated also the prognostic value of non-indexed MV tenting area in FMR and found that the cut-off value of MV tenting area predicting adverse clinical outcomes ranged from 2.5 to $3.4 \mathrm{~cm}^{2}$ (table 4$){ }^{6-9}$ Moreover, recommendations of European Association of Echocardiography defined non-indexed tenting area $>2.5 \mathrm{~cm}^{2}$ as a risk factor for recurrent MR following MV repair in FMR. ${ }^{3}$ However, 
individualised surgical treatment decisions for FMR may require adjusted MV tenting severity values to improve their prognostic relevance in the prediction of clinical outcome. Otherwise, small female or male patients (ie, BSA $1.5 \mathrm{~m}^{2}$ ) with severe FMR and a non-indexed tenting area of $2.0 \mathrm{~cm}^{2}$ may be wrongly categorised as atrial FMR type and would undergo an isolated annuloplasty despite the presence of an extensive LV remodelling.

Putting this all together, there is substantial evidence that severity of MV tenting area is associated with outcomes in patients with FMR. Adjustment of MV tenting area to BSA seems to improve its prognostic value in predicting outcomes after MV surgery. However, a prospective study is needed to further validate indexed MV tenting parameters in FMR treatment.

\section{Individualised FMR treatment based on MV tenting severity}

Current surgical FMR treatment includes MV replacement, isolated MV annuloplasty and most recently, MV annuloplasty with simultaneous subannular repair. ${ }^{8}$ Subannular MV repair techniques address mainly the papillary muscles and were developed to actively counteract $\mathrm{LV}$ remodelling in addition to annuloplasty, which corrects primarily MV annular dilation. ${ }^{20}$ However, an evidence-based treatment algorithm for FMR is still missing and it remains unclear, which patients with FMR benefit most from simultaneous subannular repair. We demonstrated that severe MV tenting, defined as MV tenting area $>1.35 \mathrm{~cm}^{2} / \mathrm{m}^{2}$, is associated with worse outcomes following an isolated MV annuloplasty. Therefore, we believe that patients with MV tenting area $>1.35 \mathrm{~cm}^{2} / \mathrm{m}^{2}$ may benefit most from additional subannular repair.

In summary, an integration of quantitative MV apparatus measurements and of validated individual factors (ie, sex, height, weight) is essential to understand the key pathogenesis in patients with FMR. Therefore, in our opinion, FMR treatment algorithm should involve indexed echocardiographic values of MV tenting area to allow personalised FMR treatment and subsequent improvement of long-term results.

\section{Limitations}

Our study possesses all the limitations of a retrospective study design. Our study is a single-centre experience, and the study population has been retrospectively analyse. Furthermore, echocardiographic variables were not validated by a core laboratory and we used 2D transthoracic echocardiography to evaluate MV tenting parameters. Therefore, the quantification of tenting volume, intercommissural MV annulus diameter and MV annular area were not possible without three-dimensional echo dataset. Although recurrent mitral regurgitation would be an interesting parameter to correlate with the BSAindexed tenting area, no systematic on-site echocardiographic follow-up was available. This is basically due to large referral area of our centre, while most patients are referred from a radius of $>100 \mathrm{~km}$. Therefore, on-site echocardiographic follow-up is often impossible due to logistic reasons. Echocardiographic follow-up was predominantly done by external outpatient cardiologists and did not include a standardised protocol to quantify recurrent MR. Last, using ROC curves for cut-off point analysis neglects time dependency of outcome events. In summary, our current findings should be confirmed by a consecutive prospective study.

\section{CONCLUSION}

Adjustment of MV tenting area to individual anatomical characteristics including body shape (BSA), mitral annular dimensions and gender provides new insights into FMR mechanisms and may thereby increase its prognostic value. In our study, BSA-adjusted MV tenting area $>1.35 \mathrm{~cm}^{2} / \mathrm{m}^{2}$ was found as a reliable echocardiographic marker which predicts reduced survival and lower freedom of adverse cardiac events following isolated MV annuloplasty. In those patients with FMR with severe MV tenting (ie, BSA-indexed MV tenting area $>1.35 \mathrm{~cm}^{2} / \mathrm{m}^{2}$ ), long-term prognosis could be potentially improved by implementing surgical strategies that specifically address LV remodelling.

Acknowledgements The authors are grateful to Bastian Geelhoed for giving advice on how to find the optimal cut-off point for the indexed values of tenting area.

Contributors The study was designed by MvS and EG. Data collection was handled by MvS, TH, FD, TS-G, JP, LM and CRS. MvS and EG did the data analyses. $\mathrm{MvS}$ and $\mathrm{EG}$ wrote the first manuscript. All authors critically revised the manuscript and agree to be accountable for all aspects of the work.

Funding The authors have not declared a specific grant for this research from any funding agency in the public, commercial or not-for-profit sectors.

Competing interests None declared.

Patient consent for publication Not required.

Ethics approval Our study protocol was approved by the local Ethics Committee (Nr. PV5382; 26th April 2017). This observational study complies with the Declaration of Helsinki.

Provenance and peer review Not commissioned; externally peer reviewed.

Data availability statement No data are available.

Open access This is an open access article distributed in accordance with the Creative Commons Attribution Non Commercial (CC BY-NC 4.0) license, which permits others to distribute, remix, adapt, build upon this work non-commercially, and license their derivative works on different terms, provided the original work is properly cited, appropriate credit is given, any changes made indicated, and the use is non-commercial. See: http://creativecommons.org/licenses/by-nc/4.0/.

ORCID iDs

Maria von Stumm http://orcid.org/0000-0001-9578-0430

Christoph R Sinning http://orcid.org/0000-0002-7331-8840

\section{REFERENCES}

1 Yiu SF, Enriquez-Sarano M, Tribouilloy C, et al. Determinants of the degree of functional mitral regurgitation in patients with systolic left ventricular dysfunction: a quantitative clinical study. Circulation 2000;102:1400-6.

2 Dudzinski DM, Hung J. Echocardiographic assessment of ischemic mitral regurgitation. Cardiovasc Ultrasound 2014;12:46.

3 Lancellotti P, Moura L, Pierard LA, et al. European association of echocardiography recommendations for the assessment of valvular regurgitation. Part 2: mitral and tricuspid regurgitation (native valve disease). Eur J Echocardiogr 2010;11:307-32. 
4 Ryan LP, Jackson BM, Parish LM, et al. Mitral valve tenting index for assessment of subvalvular remodeling. Ann Thorac Surg 2007;84:1243-9.

5 von Stumm M, Dudde F, Gasser S, et al. Prognostic value of mitral valve tenting area in patients with functional mitral regurgitation. Interact Cardiovasc Thorac Surg 2020;30:431-8.

6 Magne J, Pibarot P, Dagenais F, et al. Preoperative posterior leaflet angle accurately predicts outcome after restrictive mitral valve annuloplasty for ischemic mitral regurgitation. Circulation 2007;115:782-91.

7 Karaca O, Avci A, Guler GB, et al. Tenting area reflects disease severity and prognosis in patients with non-ischaemic dilated cardiomyopathy and functional mitral regurgitation. Eur $J$ Heart Fail 2011;13:284-91.

8 Nappi F, Lusini M, Avtaar Singh SS, et al. Risk of ischemic mitral regurgitation recurrence after combined valvular and subvalvular repair. Ann Thorac Surg 2019;108:536-43.

9 Kongsaerepong V, Shiota M, Gillinov AM, et al. Echocardiographic predictors of successful versus unsuccessful mitral valve repair in ischemic mitral regurgitation. Am J Cardiol 2006;98:504-8.

10 Gutgesell HP, Rembold CM. Growth of the human heart relative to body surface area. Am J Cardiol 1990;65:662-8.

11 Kim D-H, Heo R, Handschumacher MD, et al. Mitral valve adaptation to isolated annular dilation: insights into the mechanism of atrial functional mitral regurgitation. JACC Cardiovasc Imaging 2019;12:665-77.

12 Giustino G, Overbey J, Taylor D, et al. Sex-Based Differences in Outcomes After Mitral Valve Surgery for Severe Ischemic Mitral
Regurgitation: From the Cardiothoracic Surgical Trials Network. JACC Heart Fail 2019;7:481-90.

13 Dwivedi G, Mahadevan G, Jimenez D, et al. Reference values for mitral and tricuspid annular dimensions using two-dimensional echocardiography. Echo Res Pract 2014;1:43-50.

14 Goldstein D, Moskowitz AJ, Gelijns AC, et al. Two-year outcomes of surgical treatment of severe ischemic mitral regurgitation. $N$ Engl $J$ Med Overseas Ed 2016;374:344-53.

15 Grayburn PA, Sannino A, Packer M. Proportionate and disproportionate functional mitral regurgitation: a new conceptual framework that reconciles the results of the MITRA-FR and COAPT trials. JACC Cardiovasc Imaging 2019;12:353-62.

16 Mosteller RD. Simplified calculation of body-surface area. N Engl J Med 1987;317:1098.

17 Nishino S, Watanabe N, Kimura T, et al. Acute versus chronic ischemic mitral regurgitation: an echocardiographic study of anatomy and physiology. Circ Cardiovasc Imaging 2018;11:e007028.

18 Anwar AM, Soliman OII, ten Cate FJ, et al. True mitral annulus diameter is underestimated by two-dimensional echocardiography as evidenced by real-time three-dimensional echocardiography and magnetic resonance imaging. Int $J$ Cardiovasc Imaging 2007;23:541-7.

19 Kislitsina ON, Zareba KM, Bonow RO, et al. Is mitral valve disease treated differently in men and women? Eur J Prev Cardiol 2019;26:1433-43.

20 Harmel EK, Reichenspurner H, Girdauskas E. Subannular reconstruction in secondary mitral regurgitation: a meta-analysis. Heart 2018;104:1783-90. 